\title{
Four State Filter with Position Measurements and also with Position and Doppler Measurements
}

\author{
B.RGeetha \\ Research Scholar, Registration Number: \\ $00140811 \mathrm{~V}$, File No.:11264 \\ Department Of Computer Science \\ Pacific Academy Of Higher Education and \\ Research University, \\ Pacific Hills, Airport Road, Pratap Nagar \\ Extension, Udaipur, Rajasthan
}

\author{
J. Fazlur Rahman \\ Research Supervisor, Pacific University, \\ Director R\&D \& Head, \\ Department of Mechanical Engineering, \\ HKBK College of Engineering, Bangalore, India
}

\begin{abstract}
A one-dimensional, four state Kalman tracking filter is described for two cases: In case 1, the filter is described for position measurements only and in case 2 , the filter is described for both position and Doppler measurements. The filter estimates the range, the range-rate, the rangeacceleration and the range-jerk of a moving target such as an aircraft moving with constant jerk perturbed by a plant noise of zero mean and constant variance which accounts for maneuvers and/or other random factors. In case 1, the range coordinate of the vehicle is assumed to be measured by a track-while-scan radar sensor and incase 2 , the rangerate (Doppler) measurements are obtained by a trackwhile-scan radar sensor which employs pulsed Doppler processing such as a moving target detector providing unambiguous Doppler data [1,2]. The measurements are obtained at uniform sampling intervals of time $\mathrm{T}$ seconds and all measurements are noisy. The filter structures have been defined and the steady state results are given for both cases.
\end{abstract}

\section{INTRODUCTION}

Consider a constant jerk filter[3,4] for tracking an aircraft moving with a constant jerk perturbed by a plant noise of zero mean and constant variance. The filter estimates the optimum range, range-rate, range-acceleration and rangejerk. The position coordinate of the vehicle is assumed to be measured by a track-while-scan radar sensor at uniform sampling intervals of time $T$ seconds through random noise [5-7]..

\section{DYNAMIC MODEL}

The dynamics of the target is assumed to be described by the vector matrix equation of the form

$$
X_{n+1}=F X_{n}+W_{n}
$$

$$
\text { where } \quad X_{n}=\left[\begin{array}{c}
x_{n} \\
\dot{x}_{n} \\
\ddot{x}_{n} \\
\dddot{x}_{n}
\end{array}\right]
$$

$x_{n}, \dot{x}_{n}, \ddot{x}_{n}$ and $\dddot{x}_{n}$ are the vehicle position, velocity, acceleration and jerk respectively at scan $n, T$ is the sampling time.

$$
\begin{gathered}
F=\left[\begin{array}{cccc}
1 & T & T^{2} / 2 & T^{3} / 6 \\
0 & 1 & T & T^{2} / 2 \\
0 & 0 & 1 & T \\
0 & 0 & 0 & 1
\end{array}\right] \\
W_{n}=\left[\begin{array}{c}
0 \\
0 \\
0 \\
w_{n}
\end{array}\right]
\end{gathered}
$$

$W_{n}$ is the plant noise vector with covariance matrix given by

$$
Q=\left[\begin{array}{cccc}
0 & 0 & 0 & 0 \\
0 & 0 & 0 & 0 \\
0 & 0 & 0 & 0 \\
0 & 0 & 0 & \sigma_{j}^{2}
\end{array}\right]
$$

\section{CASE 1: MEASUREMENT EQUATION FOR RANGE MEASUREMENTS ONLY}

The measurement equation may be written as

$$
y_{n}=H X_{n}+v_{n}
$$

$y_{n}$ is the measured position at scan $n$ and $v_{n}$ is the random noise corrupting the measurement at scan $n$ and

$$
H=\left[\begin{array}{llll}
1 & 0 & 0 & 0
\end{array}\right]
$$

The statistical properties of the measurement noise are assumed to be:

$$
\begin{aligned}
& E\left\{v_{n}\right\}=0 \\
& E\left\{v_{n}^{2}\right\}=\sigma_{x}^{2}=\text { constant for all } n
\end{aligned}
$$




$$
E\left\{v_{n} v_{K}\right\}=0 \quad \text { for } n \neq k
$$

\section{CASE 2: MEASUREMENT EQUATION FOR BOTH RANGE AND RANGE-RATE MEASUREMENTS}

The measurement model is assumed to be given by

$$
Z_{n}=H X_{n}+V_{n}
$$

where

$$
\begin{aligned}
Z_{n} & =\left[\begin{array}{l}
x_{m}(n) \\
\dot{x}_{m}(n)
\end{array}\right] \\
H & =\left[\begin{array}{llll}
1 & 0 & 0 & 0 \\
0 & 1 & 0 & 0
\end{array}\right] \\
V_{n} & =\left[\begin{array}{l}
v_{x}(n) \\
v_{y}(n)
\end{array}\right] \\
R & =\left[\begin{array}{cc}
\sigma_{x}^{2} & 0 \\
0 & \sigma_{d}^{2}
\end{array}\right]
\end{aligned}
$$

$x_{m}(n)$ is the measured radial range at scan $n, \dot{x}_{m}(n)$ is the measured range rate at scan $n, R$ is the covariance matrix of the measurement noise. The maneuver noise $W_{n}$ is assumed to be independent of the measurement noise $V$.

\section{FILTERING EQUATIONS}

Applying Kalman filtering algorithm to the problem, the optimum estimate of the state vector is given by

$$
\hat{X}_{n}=\tilde{X}_{n}+K_{n}\left[y_{n}-H \tilde{X}_{n}\right]
$$

with

$$
\tilde{X}_{n+1}=F \hat{X}_{n}
$$

$K_{n}=\tilde{P}_{n} H^{T}\left(H \tilde{P}_{n} H^{T}+R\right)^{-1}$

$\widetilde{P}_{n+1}=F \hat{P}_{n} F^{T}+Q$

$$
\hat{P}_{n}=\left(I-K_{n} H\right) \tilde{P}_{n}
$$

$\hat{X}_{n}$ is the optimum estimate of the state vector after the measurement is processed, $\tilde{X}_{n}$ is the optimum estimate of the state vector before the measurement is processed,
$K_{n}$ is the Kalman gain vector, $\hat{P}_{n}$ is the covariance matrix of estimation errors after the measurements are processed and $\widetilde{P}_{n}$ is the covariance matrix of estimation errors before the measurements are processed. $R=\sigma_{x}^{2}$ is the variance of the measurement noise.

\section{STEADY STATE ANALYSIS}

In the steady state,

$$
\begin{gathered}
\widetilde{P}_{n+1}=\widetilde{P}_{n}=\tilde{P} \quad \text { Say } \\
\hat{P}_{n+1}=\hat{P}_{n}=\hat{P} \\
K_{n+1}=K_{n}=K
\end{gathered}
$$

Hence, in the steady state, $13-15$ may be written as

$$
\begin{aligned}
& K=\widetilde{P} H^{T}\left(H \tilde{P} H^{T}+R\right)^{-1} \\
& \tilde{P}=F \hat{P} F^{T}+Q \\
& \hat{P}=(I-K H) \tilde{P}
\end{aligned}
$$

17 may equivalently be expressed as

$$
K=\hat{P} H^{T} R^{-1}
$$

\section{NUMERICAL RESULTS}

The normalized gains and covariances are evaluated for the following values of the parameters and the computer results are tabulated separately for range measurements only case and for both range and range-rate measurements only case:

Parameters:

$$
\begin{aligned}
T & =4 \text { seconds } \\
\sigma_{x} & =0.0509 \mathrm{Km} \\
\sigma_{j} & =0.00190875 \mathrm{Km} / \mathrm{s}^{3}
\end{aligned}
$$

Computer Results for range measurements only Case 1 Executing the recursive Kalman filter matrix equations 17 to 19 to the steady state (about 500 iterations, we get the steady state predicted covariance matrix as

$$
\tilde{P}=\left[\begin{array}{llll}
0.5576 & 0.3746 & 0.1529 & 0.0312 \\
0.3746 & 0.2728 & 0.1193 & 0.0263 \\
0.1529 & 0.1193 & 0.0570 & 0.0141 \\
0.0312 & 0.0263 & 0.0141 & 0.0058
\end{array}\right]
$$

The steady state filtered covariance matrix is obtained as 


$$
\hat{P}=\left[\begin{array}{llll}
0.0245 & 0.0164 & 0.0067 & 0.0014 \\
0.0164 & 0.0321 & 0.0211 & 0.0063 \\
0.0067 & 0.0211 & 0.0169 & 0.0059 \\
0.0014 & 0.0063 & 0.0059 & 0.0041
\end{array}\right]
$$

Comparing 21 and 22, it may be noted that the covariances of estimation errors go down by incorporating the measurement into the tracking process.

The steady state Kalman gain matrix is obtained as

$$
K=\left[\begin{array}{l}
0.9561 \\
0.6423 \\
0.2622 \\
0.0535
\end{array}\right]
$$

Computer Results for range and range-rate measurements only case 2

Parameters

$$
\begin{aligned}
& T=2 \text { seconds } \\
& \sigma_{x}=0.1600 \mathrm{Km} \\
& \sigma_{j}=0.0409 \mathrm{Km} / \mathrm{sec} \text { onds }^{3} \\
& \sigma_{d}=0.2600
\end{aligned}
$$

\section{Computer Results}

Executing the recursive Kalman filter matrix equations 17 to 19 to the steady state (about 500 iterations with the measurement matrix and the covariance matrix of measurement errors given by 10 , we get the steady state predicted covariance matrix as

$$
\tilde{P}=\left[\begin{array}{llll}
0.3810 & 0.2576 & 0.1078 & 0.0229 \\
0.2576 & 0.1946 & 0.0889 & 0.0207 \\
0.1078 & 0.0889 & 0.0451 & 0.0119 \\
0.0229 & 0.0207 & 0.0119 & 0.0053
\end{array}\right] \text {. }
$$

The steady state filtered covariance matrix is obtained as

$$
\hat{P}=\left[\begin{array}{llll}
0.0213 & 0.0111 & 0.0034 & 0.0004 \\
0.0111 & 0.0214 & 0.0141 & 0.0042 \\
0.0034 & 0.0141 & 0.0122 & 0.0045 \\
0.0004 & 0.0042 & 0.0045 & 0.0037
\end{array}\right]
$$

The steady state Kalman gain matrix is obtained
As $K=\left[\begin{array}{ll}0.8332 & 0.1639 \\ 0.4328 & 0.3170 \\ 0.1330 & 0.2085 \\ 0.0167 & 0.0624\end{array}\right]$

Comparing 21 and 24, it may be noted that the predicted covariances of estimation errors before the measurement is processed go down as a result of incorporating the rangerate measurement also into the tracking process, thus providing better tracking accuracies. Again, comparing 22 and 25 , it may be noted that the filtered covariances of estimation errors after the measurement is incorporated, go down as a result of incorporating the range-rate measurement also into the tracking process, thus providing improved tracking accuracies. The results of case 2 degenerate to those of case 1 when the Doppler variance is zero.

\section{REFERENCES}

[1] F.R.Castella, Tracking Accuracies with Position and Rate Measurements, IEEE Transactions on Aerospace and Electronic Systems, AES-17, pp 433-437, May 1981

[2] K.V.Ramachandra, Kalman Filtering Techniques for Radar Tracking, Marcel-Dekker, Inc., New York, 2000

[3] K.V.Ramachandra and C.Ramesh, Analytical steady state results for a four state Constant jerk filter, In Proceedings of International Radar Symposium, India (IRSI-2001) held in Bangalore, India during 11-14, December 2001, pp 306-25

[4] K.V.Ramachandra and B.R.Mohan, Analytical results of some higher order Kalman tracking filters, In Proceedings of International Radar Symposium, India, (IRSI - 2003) held in Bangalore, India during 2-5, December 2003, pp 666-681.

[5] B.R.Geetha, Discrete and Continuous-Time Kalman Filters for Computer Applications, International Journal for Computer Applications, Pp 102-108, Issue 3, Volume1, February2013, ISSN-2250-1797. Available on line on http://www.rspublication.com/ijca/ijca-index.htm,

[6] B.R.Geetha and K.V.Ramachandra A Three State Kalman Filter with Range and Range-Rate Measurements, International Journal for Computer Applications, Pp. 85 - 101, Volume 1, February 2013 ISSN-2250-1797. Available on line on http://www.rspublication.com/ijca/ijca-index.htm,

[7] K.V.Ramachandra, B.R.Mohan and B.R.Geetha, A three state Kalman tracker using position and rate measurement, IEEE Transactions on Aerospace and Electronic Systems, AES-29, No.1, Pp.215-222, January 1993 